\title{
Regulation of Cooling Mode of Thermoelectric Bloc
}

\author{
Evgeniy N. Vasil'ev* \\ Institute of Computational Modelling SB RAS \\ Krasnoyarsk, Russian Federation
}

Received 06.04.2021, received in revised form 11.05.2021, accepted 21.06.2021

\begin{abstract}
With the help of a computational model, the cooling modes of a block of a thermoelectric refrigeration unit designed for ship's provision and freezing chambers are studied. The computational model, based on the numerical solution of a system of nonlinear algebraic equations, takes into account the operating characteristics of a serial thermoelectric module and the thermal resistances of heat removal and supply devices. The dependences of the cooling power and the coefficient of performance of the block on the supply current of the thermoelectric modules are calculated. The analysis of the cooling modes of the thermoelectric block is carried out and recommendations for regulating the current strength depending on the current temperature conditions in the provision and freezing chambers are developed.
\end{abstract}

Keywords: thermoelectric module, cooling power, coefficient of performance, heat exchange, thermal conductivity.

\section{Регулирование режима охлаждения термоэлектрического блока}

Е.Н. Васильев

Институт вычислительного моделирования СО РАН Российская Федерачия, Красноярск

Аннотация. С помощью вычислительной модели исследованы режимы охлаждения блока термоэлектрической холодильной установки, предназначенной для судовых провизионной и морозильной камер. Вычислительная модель, основанная на численном решении системы нелинейных алгебраических уравнений, учитывает рабочие характеристики серийного

(C) Siberian Federal University. All rights reserved

This work is licensed under a Creative Commons Attribution-Non Commercial 4.0 International License (CC BY-NC 4.0).

* Corresponding author E-mail address: ven@icm.krasn.ru 
термоэлектрического модуля и термические сопротивления устройств отвода и подвода теплоты. Рассчитаны зависимости холодопроизводительности и холодильного коэффициента блока от силы тока питания термоэлектрических модулей. Проведен анализ режимов охлаждения термоэлектрического блока и выработаны рекомендации по регулированию величины силы тока в зависимости от текущих температурных условий в провизионной и морозильной камерах.

Ключевые слова: термоэлектрический модуль, холодопроизводительность, холодильный коэффициент, теплообмен, теплопроводность.

Цитирование: Васильев, Е.Н. Регулирование режима охлаждения термоэлектрического блока / Е.Н. Васильев // Журн. Сиб. федер. ун-та. Техника и технологии, 2021, 14(4). С. 416-423. DOI: 10.17516/1999-494X-0322

\section{Введение}

Термоэлектрические устройства активно применяются в современной технике для охлаждения как миниатюрных объектов, так и холодильных камер большого объема $[1,2]$. Преимуществом термоэлектрического способа охлаждения по сравнению с традиционным компрессорным методом является компактность, надежность, экологичность и бесшумность, основной недостаток - сравнительно низкий холодильный коэффициент. В работах $[3,4]$ представлена оригинальная конструкция блока термоэлектрической холодильной установки (ТЭХУ) для провизионных и морозильных камер корабельного назначения. В конструкции блока ТЭХУ использованы технические решения, обеспечивающие повышение эффективности охлаждения за счет снижения температурных перепадов на термических сопротивлениях элементов конструкции и максимальное использование потенциала существующих термоэлектрических модулей (ТЭМ). В настоящей работе проведен расчет и анализ характеристик процесса охлаждения с целью выбора оптимальных режимов работы блока ТЭХУ в зависимости от текущих значений температуры в провизионной и морозильной камерах.

\section{1. Описание и характеристики блока}

\section{термоэлектрической холодильной установки}

Предложенная в [3] конструкция ТЭХУ имеет блочную структуру, при этом полагается, что провизионные и морозильные камеры будут оснащены однотипными блоками охлаждения. Каждый блок содержит в себе восемь термоэлектрических модулей, к каждому из которых присоединены устройства подвода и отвода теплоты. Устройство подвода теплоты осуществляет теплопередачу из охлаждаемого объема к холодной стороне ТЭМ и состоит из воздушного пластинчатого радиатора и термосифонов. От горячей стороны ТЭМ теплота отводится с помощью жидкостного теплообменника, охлаждаемого забортной водой.

Охлаждение камер обеспечивают термоэлектрические модули, создающие отрицательный температурный перепад на своих границах. Процесс теплопередачи в устройствах подвода и отвода теплоты сопровождается потерями температурного перепада, снижающими эффективность охлаждения блока ТЭХУ. Величина потерь в устройствах подвода и отвода теплоты прямо пропорциональна их термическим сопротивлениям. Исследование влияния термических сопротивлений на характеристики термоэлектрического охлаждения было проведено в работах [5-8]. Одним из путей повышения эффективности блока ТЭХУ является оптимизация режимов его работы. Регулирование работы блока осуществляется с помощью 
управления силой тока питания ТЭМ, которая определяет основные характеристики процесса охлаждения.

Основными характеристиками, определяющими выбор режимов работы блока ТЭХУ, являются холодопроизводительность $Q$ и холодильный коэффициент $\varepsilon$. Холодопроизводительность характеризует мощность охлаждения, а холодильный коэффициент определяет экономичность процесса. Холодильный коэффициент равен отношению холодопроизводительности блока к потребляемой им электрической мощности $W$ :

$$
\varepsilon=\frac{Q}{W} .
$$

Холодильный коэффициент блока ТЭХУ зависит от эффективности ТЭМ, характеризуемой величиной COP (coefficient of performance), также на него оказывают влияние потери температурного перепада в устройствах подвода и отвода теплоты.

В компрессорных холодильниках распространена релейная схема управления режимом охлаждения, когда холодильный агрегат периодически включается и выключается для поддержания заданной температуры в объеме камеры. Такая схема управления неприемлема для блока ТЭХУ, поскольку ТЭМ имеют ограниченный ресурс на количество циклов «включениевыключение». Поэтому управление режимом охлаждения блока ТЭХУ должно производиться путем плавного изменения силы тока питания ТЭМ. Для оптимального управления режимами необходимо установить зависимости характеристик охлаждения от силы тока с учетом значения температуры в продуктовых камерах.

Расчет характеристик охлаждения блока ТЭХУ проводили с учетом рабочих характеристик ТЭМ с помощью соотношения, связывающего общий температурный перепад $\Delta T_{e}=T_{1}-T_{0}$ с перепадами температур на отдельных элементах блока:

$$
\Delta T_{e}=R_{T} Q+R_{s}(Q+W)-\Delta T_{\text {ТЭм }}=\left(R_{s}+R_{T}\right) Q+R_{s} U(I, Q) I-\Delta T_{\text {Тэм }}(I, Q) .
$$

Здесь $T_{1}, T_{0}$ - значения температуры воздуха в камере и забортной воды, $\Delta T_{\text {Тэм }}-$ перепад температуры между горячей и холодной сторонами ТЭМ, $U, I-$ напряжение и сила тока электропитания ТЭМ. Рабочие характеристики термоэлектрического модуля $Q\left(\Delta T_{\mathrm{Tэм}}\right)$ и $U\left(\Delta T_{\text {Тэм }}\right)$ являются исходными данными для определения зависимостей $U(I, Q)$ и $\Delta T_{\text {тэм }}(I, Q)$ с помощью интерполяционных многочленов, методика расчета этих зависимостей приведена в [5]. Решение нелинейной алгебраической системы уравнений для заданных значений $R_{S}, R_{T}, \Delta T_{e}$ и $I$ позволяет рассчитать величины $Q, W$ и $\varepsilon$.

Исследование режимов охлаждения провизионной и морозильной камер основано на анализе зависимостей $Q(I)$ и $\varepsilon(I)$. Расчет этих зависимостей производили для значений температуры $T_{p}=3{ }^{\circ} \mathrm{C}$ в провизионной камере и $\mathrm{T}_{f}=$ минус $18^{\circ} \mathrm{C}$ в морозильной камере, температуры забортной воды $T_{0}=20^{\circ} \mathrm{C}$. Термические сопротивления воздушного и жидкостного теплообменников предварительно были определены из численного моделирования по методике [9], термическое сопротивление термосифонов было установлено в экспериментах [4]. При этом характерное значение термического сопротивления для устройства теплоподвода не превышают $0,5 \mathrm{~K} / \mathrm{BT}$, для устройства теплоотвода $-0,1$ К/Вт. Для анализа влияния термических сопротивлений устройств подвода и отвода теплоты на характеристики охлаждения были проведены расчеты при значениях $R_{T}=0,4$ и $0,5 \mathrm{~K} / \mathrm{BT}, R_{S}=0,08$ и $0,1 \mathrm{~K} / \mathrm{B}$, имеющих одинаковую относительную 
разницу между собой. Расчеты и анализ осуществлены для серийного термоэлектрического модуля «S-199-14-11» с максимальными значениями температурного перепада $\Delta T_{\mathrm{T} э \mathrm{M}}=72,5^{\circ} \mathrm{C}$ и холодопроизводительности $Q=124,2 \mathrm{~W}$ при максимальной силе тока $I_{\max }=7,9 \mathrm{~A}$.

Выбор величины силы тока питания ТЭМ в зависимости от текущей температуры в провизионной и морозильной камерах является основной задачей регулирования, поскольку именно от силы тока зависят характеристики охлаждения блока ТЭХУ. Производители термоэлектрических систем в сопроводительной документации обычно предлагают в качестве оптимального параметра электропитания ТЭМ уровень 0.75 от максимальных значений тока или напряжения без учета специфики режимов охлаждения и термических сопротивлений. Зависимости $\varepsilon(I)$ и $Q(I)$, рассчитанные с учетом термических сопротивлений устройств подвода и отвода теплоты, служат основой для выработки алгоритма оптимального управления величиной силы тока с целью поддержания заданных значений температуры в провизионной и морозильной камерах.

\section{2. Регулирование режима охлаждения в провизионной камере}

Для температурных условий в провизионной камере $\left(T_{p}=3{ }^{\circ} \mathrm{C}\right)$ зависимости $Q(I)$ рассчитаны для одного ТЭМ и изображены на рис. 1. Графики $Q(I)$ соответствуют следующим значениям: $\left.R_{T}=0,5 \mathrm{~K} / \mathrm{Bт}, R_{S}=0,1 \mathrm{~K} / \mathrm{Bт} \mathrm{(линия} 1\right), R_{T}=0,4 \mathrm{~K} / \mathrm{Bт}, R_{S}=0,1 \mathrm{~K} / \mathrm{BT}$ (линия 2$), R_{T}=0,5 \mathrm{~K} / \mathrm{BT}$, $R_{S}=0,08 \mathrm{~K} / \mathrm{BT}$ (линия 3). Зависимости $Q(I)$ принимают положительное значение, начиная со значения $I_{1} \approx 0,8$ А, также они характеризуются наличием максимумов, отличающихся расположением и величиной. Зависимости ходильного коэффициента $\varepsilon(I)$ для провизионной камеры приведены на рис. 2. Зависимости $\varepsilon(I)$ тоже принимают положительное значение при $I>0,8$ А и имеют максимумы при сравнительно низких значениях силы тока $I_{2}=1,6-1,7 \mathrm{~A}$.

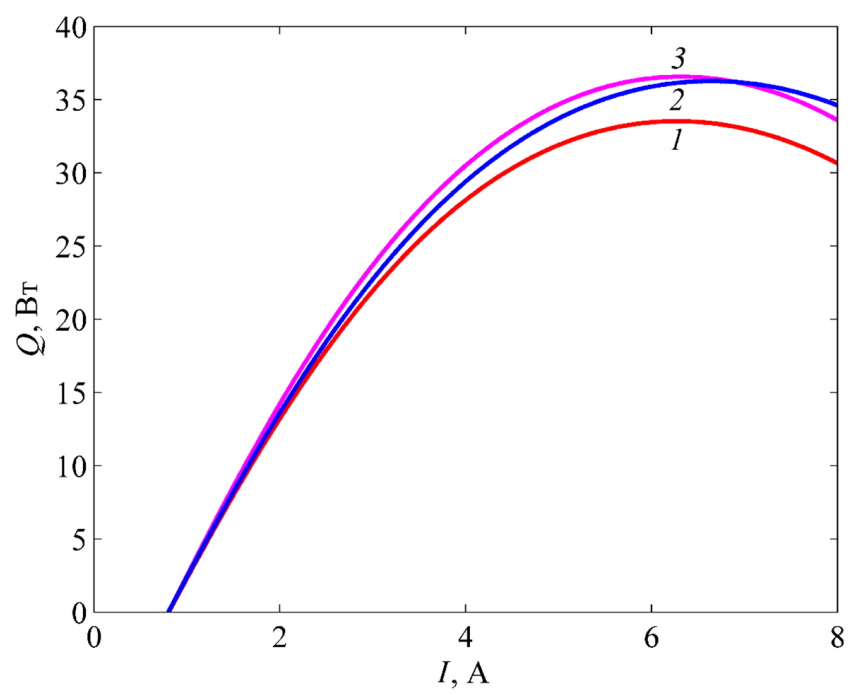

Рис. 1. Зависимости холодопроизводительности $Q(I)$ для провизионной камеры при следующих значениях термических сопротивлений: $1-R_{T}=0,5 \mathrm{~K} / \mathrm{BT}, R_{S}=0,1 \mathrm{~K} / \mathrm{BT} ; 2-R_{T}=0,4 \mathrm{~K} / \mathrm{BT}, R_{S}=0,1 \mathrm{~K} / \mathrm{BT}$; $3-R_{T}=0,5 \mathrm{~K} / \mathrm{BT}, R_{S}=0,08 \mathrm{~K} / \mathrm{BT}$

Fig. 1. Dependences of cooling power $Q(I)$ for a provision chamber with the following values of thermal resistance: $1-R_{T}=0,5 \mathrm{~K} / \mathrm{W}, R_{S}=0,1 \mathrm{~K} / \mathrm{W} ; 2-R_{T}=0,4 \mathrm{~K} / \mathrm{W}, R_{S}=0,1 \mathrm{~K} / \mathrm{W} ; 3-R_{T}=0,5 \mathrm{~K} / \mathrm{W}, R_{S}=0,08 \mathrm{~K} / \mathrm{W}$ 


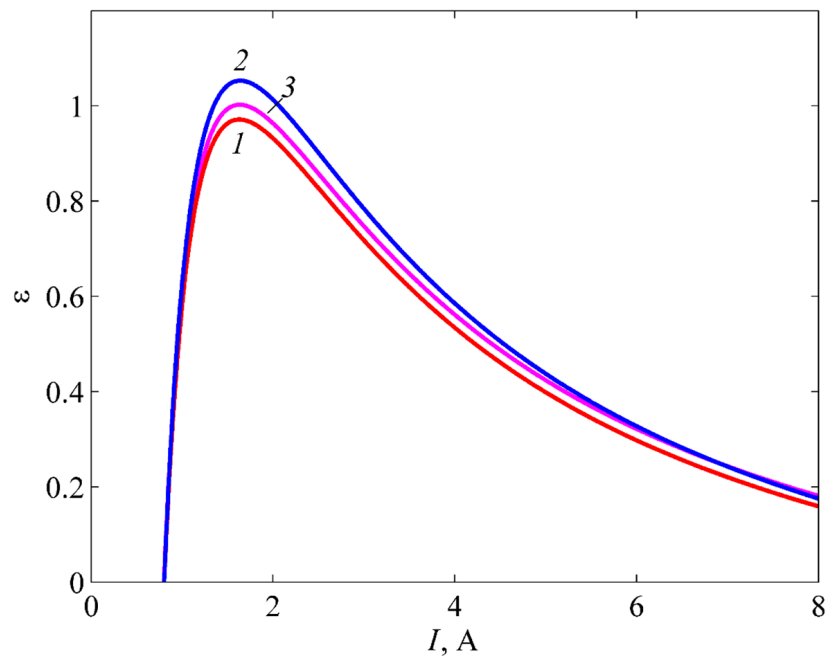

Рис. 2. Зависимости холодильного коэффициента $\varepsilon(I)$ для провизионной камеры при следующих значениях термических сопротивлений: $1-R_{T}=0,5 \mathrm{~K} / \mathrm{BT}, R_{S}=0,1 \mathrm{~K} / \mathrm{BT} ; 2-R_{T}=0,4 \mathrm{~K} / \mathrm{BT}, R_{S}=0,1 \mathrm{~K} / \mathrm{BT}$; $3-R_{T}=0,5 \mathrm{~K} / \mathrm{BT}, R_{S}=0,08 \mathrm{~K} / \mathrm{BT}$

Fig. 2. Dependences of cooling coefficient $\varepsilon(I)$ for a provision chamber with the following values of thermal resistance: $1-R_{T}=0,5 \mathrm{~K} / \mathrm{W}, R_{S}=0,1 \mathrm{~K} / \mathrm{W} ; 2-R_{T}=0,4 \mathrm{~K} / \mathrm{W}, R_{S}=0,1 \mathrm{~K} / \mathrm{W} ; 3-R_{T}=0,5 \mathrm{~K} / \mathrm{W}, R_{S}=0,08 \mathrm{~K} / \mathrm{W}$

Проведем анализ режимов охлаждения при значениях термического сопротивления $R_{T}=0,4 \mathrm{~K} / \mathrm{B}$ и $R_{S}=0,1 \mathrm{~K} / \mathrm{Bт} \mathrm{для} \mathrm{провизионной} \mathrm{камеры,} \mathrm{в} \mathrm{которой} \mathrm{должна} \mathrm{поддерживаться}$ заданная температура $T_{p}=3{ }^{\circ} \mathrm{C}\left(\Delta T_{e}=\right.$ минус $\left.17^{\circ} \mathrm{C}\right)$. Во всем диапазоне возможных значений силы тока (0-7,9 А) для рассматриваемого ТЭМ выделим три характерных значения. При силе тока, превышающей значение $I_{1}=0,8 \mathrm{~A}$, зависимость $Q(I)$ принимает положительные значения (рис. 1, линия 2). Холодильный коэффициент достигает максимального значения $\varepsilon_{\max }=1,05$ при $I_{2}=1,64 \mathrm{~A}$ (рис. 2). Режиму максимальной холодопроизводительности соответствует значение силы тока $I_{3}=6,31 \mathrm{~A}$, при этом для одного ТЭМ $Q_{\max }=36,6$ Вт, а суммарная холодопроизводительность блока составляет 292,8 Вт. Интервал значений $I_{1}-I_{3}$ определяет рабочий диапазон токов питания ТЭМ, поскольку при значениях $I>I_{3}$ холодопроизводительность ТЭМ снижается в сочетании с одновременным ростом потребляемой мощности, а при $I<I_{1}$ охлаждение вообще сменяется на нагрев.

Выбор величины тока производится в диапазоне $I_{1}-I_{3}$ и зависит от среднего значения температуры в провизионной камере. Режим максимальной холодопроизводительности требует самых высоких значений тока и потребляемой мощности. Такой режим должен задаваться только в случае, когда необходимо наиболее интенсивное охлаждение объема провизионной камеры, например при загрузке новой партии продуктов. В обычных условиях для эксплуатации блока ТЭХУ целесообразны более экономичные режимы работы. Пологий характер зависимости $Q(I)$ позволяет существенно повысить экономичность охлаждения при незначительном снижении холодопроизводительности. Так, величина $Q=0,9, Q_{\max }=32,9$ Вт обеспечивается при силе тока $I=4,36$ А, при этом значение холодильного коэффициента возрастет на 77 \% (с 0,3 при $I=6,31$ А до 0,53$)$. Температурные условия в объеме провизионной камеры характеризуются величиной $\Delta T_{p}=T_{1}-T_{p}$, определяющей отклонение текущей средней температуры от заданного 
значения $\mathrm{T}_{p}=3{ }^{\circ} \mathrm{C}$. По мере уменьшения $\Delta T_{p}$ силу тока следует плавно уменьшать до значения $I_{2}=1,64 \mathrm{~A}$, соответствующего режиму работы блока с максимальным холодильным коэффициентом. Этот режим оптимален при малых положительных значениях $\Delta T_{p} \approx 0-2{ }^{\circ} \mathrm{C}$, величина этого интервала может меняться в зависимости от технических требований к провизионной камере. Близкие к нулю и отрицательные температуры недопустимы для хранения многих продуктов (овощи, фрукты и др.), поэтому при достижении значения температуры $\mathrm{T}_{1}=\mathrm{T}_{p}$ величина силы тока должна быть уменьшена вплоть до значения $I_{1}$, при котором дальнейшее охлаждение и снижение температуры в провизионной камере прекращается.

\section{3. Регулирование режима охлаждения в морозильной камере}

Морозильная камера, в которой поддерживается температура $\mathrm{T}_{f}=$ минус $18{ }^{\circ} \mathrm{C}$, характеризуется существенно большей температурной разницей $\Delta T_{e}=$ минус $38{ }^{\circ} \mathrm{C}$, что обуславливает снижение как холодопроизводительности блока, так и его холодильного коэффициента. На рис. 3 приведены зависимости $Q(I)$, рассчитанные для одного ТЭМ при различных значениях термических сопротивлений $R_{T}$ и $R_{S}$. В целом характер зависимостей $Q(I)$ для морозильной камеры имеет аналогичный вид, однако уровень значений холодопроизводительности снизился в 1,5-2 раза по сравнению с провизионной камерой. Положительные значения зависимости $Q(I)$ на рис. 3 принимают, начиная с более высокого значения силы тока $(I \approx 2,2 \mathrm{~A})$. Зависимости ходильного коэффициента $\varepsilon(I)$ для морозильной камеры приведены на рис. 4. Максимальные значения $\varepsilon$ для морозильной камеры примерно в 4 раза ниже, чем для провизионной, а сами максимумы достигаются при более высоких значениях силы тока.

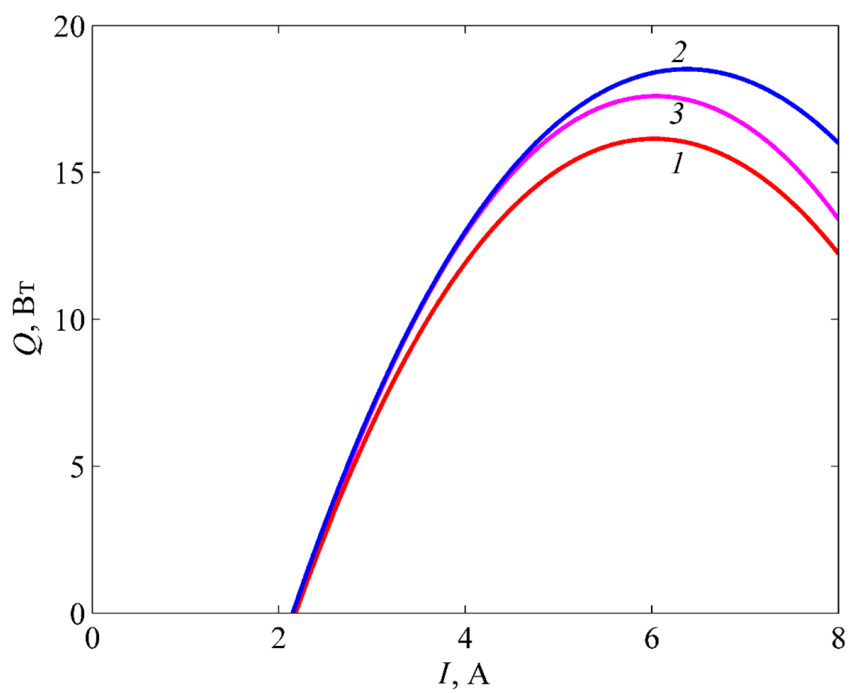

Рис. 3. Зависимости холодопроизводительности $Q(I)$ для морозильной камеры при следующих значениях термических сопротивлений: $1-R_{T}=0,5 \mathrm{~K} / \mathrm{BT}, R_{S}=0,1 \mathrm{~K} / \mathrm{BT} ; 2-R_{T}=0,4 \mathrm{~K} / \mathrm{BT}, R_{S}=0,1 \mathrm{~K} / \mathrm{BT} ; 3-$ $R_{T}=0,5 \mathrm{~K} / \mathrm{BT}, R_{S}=0,08 \mathrm{~K} / \mathrm{BT}$

Fig. 3. Dependences of cooling power $Q(I)$ for a freezing chamber with the following values of thermal resistance: $1-R_{T}=0,5 \mathrm{~K} / \mathrm{W}, R_{S}=0,1 \mathrm{~K} / \mathrm{W} ; 2-R_{T}=0,4 \mathrm{~K} / \mathrm{W}, R_{S}=0,1 \mathrm{~K} / \mathrm{W} ; 3-R_{T}=0,5 \mathrm{~K} / \mathrm{W}, R_{S}=0,08 \mathrm{~K} / \mathrm{W}$ 


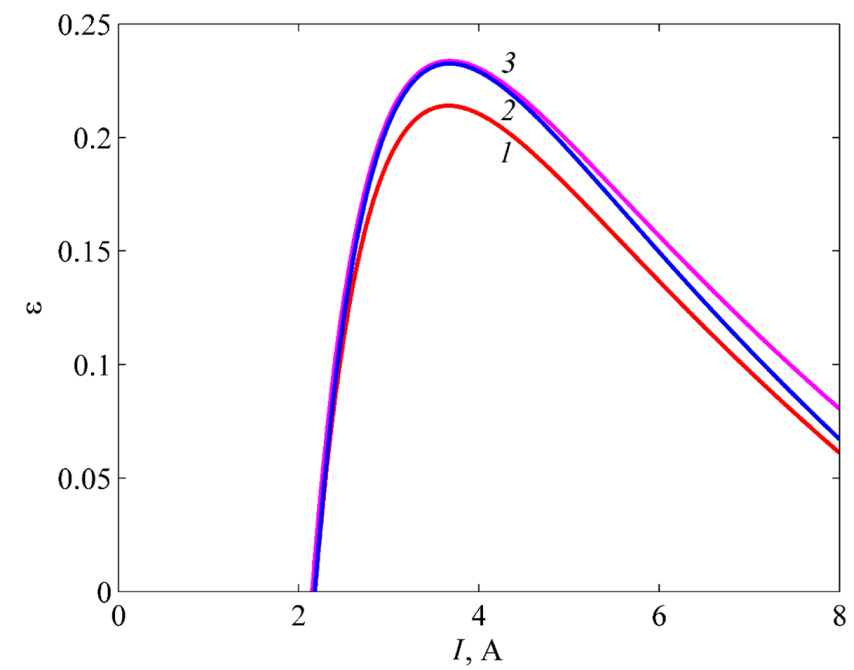

Рис. 4. Зависимости холодильного коэффициента $\varepsilon(I)$ для морозильной камеры при следующих значениях термических сопротивлений: $1-R_{T}=0,5 \mathrm{~K} / \mathrm{BT}, R_{S}=0,1 \mathrm{~K} / \mathrm{BT} ; 2-R_{T}=0,4 \mathrm{~K} / \mathrm{BT}, R_{S}=0,1 \mathrm{~K} / \mathrm{BT}$; $3-R_{T}=0,5 \mathrm{~K} / \mathrm{BT}, R_{S}=0,08 \mathrm{~K} / \mathrm{BT}$

Fig. 4. Dependences of cooling coefficient $\varepsilon(I)$ for a freezing chamber with the following values of thermal resistance: $1-R_{T}=0,5 \mathrm{~K} / \mathrm{W}, R_{S}=0,1 \mathrm{~K} / \mathrm{W} ; 2-R_{T}=0,4 \mathrm{~K} / \mathrm{W}, R_{S}=0,1 \mathrm{~K} / \mathrm{W} ; 3-R_{T}=0,5 \mathrm{~K} / \mathrm{W}, R_{S}=0,08 \mathrm{~K} / \mathrm{W}$

Анализ режимов охлаждения морозильной камеры также проведен для значений термического сопротивления $R_{T}=0,4 \mathrm{~K} / \mathrm{Bт} \mathrm{и} R_{S}=0,1 \mathrm{~K} / \mathrm{B}$. Характерные величины силы тока $I_{1}, I_{2}$ и $I_{3}$ для условий работы блока в морозильной камере имеют более высокие значения. Режим охлаждения реализуется при $I>I_{1}=2,18 \mathrm{~A}$, максимум холодильного коэффициента $\varepsilon_{\max }=0,23$ достигается при $I_{2}=3,67 \mathrm{~A}$ (рис. 4 , линия 2), максимальная холодопроизводительность для отдельного ТЭМ $Q_{\max }=17,6$ Вт при $I_{3}=6,04$ А (рис. 3 , линия 2), а суммарная холодопроизводительность блока при этом равна 140,8 Вт. Режим максимальной холодопроизводительности требует наиболее высоких затрат энергии и характеризуется сравнительно низким холодильным коэффициентом 0,15 . Такой режим целесообразен при больших положительных отклонениях средней температуры в объеме камеры от заданного значения $\mathrm{T}_{f}=$ минус $18{ }^{\circ} \mathrm{C}$. Более экономичному режиму работы ТЭМ без существенного снижения холодопроизводительности соответствует значение силы тока $I=4,78 \mathrm{~A}$, определенное по уровню $Q=0,9, Q_{\max }=15,84 \mathrm{~B}$, холодильный коэффициент в этом случае увеличивается на 37 \% до 0,2. Работа термоэлектрического блока при температуре в камере, близкой к заданному значению $\mathrm{T}_{f}$, оптимальна при силе тока $I_{2}$, когда обеспечивается максимальный холодильный коэффициент.

При достижении заданного значения $\mathrm{T}_{f}$ возможны два варианта работы термоэлектрического блока. В первом варианте устанавливается сила тока $I=I_{1}$, в данном режиме охлаждение камеры прекратится $(Q=0)$, а энергопотребление блока будет на минимальном уровне. В втором варианте работа блока продолжается при $I=I_{2}$ с возможностью дальнейшего снижения температуры. Для морозильной камеры дополнительное снижение температуры не является негативным фактором, поскольку при этом улучшается сохранность продуктов и увеличивается длительность их хранения. Выбор варианта работы блока при значениях текущей температуры $T_{1} \geq \mathrm{T}_{f}$ должен задаваться техническими требованиями к морозильной камере. 


\section{Заключение}

Рассчитаны зависимости $Q(I)$ и $\varepsilon(I)$ с учетом термических сопротивлений устройств подвода и отвода теплоты термоэлектрического блока охлаждения. По полученным зависимостям определены значения силы тока, при которых реализуются режимы максимального холодильного коэффициента, а также минимальной и максимальной холодопроизводительности. Анализ режимов блока ТЭХУ был направлен на выбор оптимальной величины силы тока в зависимости от температурных условий в провизионной и морозильной камерах с целью поддержания заданной температуры и повышения эффективности охлаждения.

\section{Список литературы / References}

[1] Zhao D., Tan G. A review of thermoelectric cooling: materials, modeling and applications, Applied Thermal Engineering, 2014, 66, 15-24.

[2] Анатычук Л.И., Вихор Л.Н., Николаенко Ю.Е., Рассамакин Б. М., Розвер Ю. Ю. Крупноформатный термоэлектрический модуль охлаждения с тепловыми трубами, Термоэлектричество, 2011, (1), 53-59 [Anatychuk L.I., Vichor L.N., Nikolaenko Yu. Ye. Rassamakin B.M., Rozver Yu. Yu. Largesized thermoelectric module with heat pipes. Journal of Thermoelectricity, 2011, (1), 50-55].

[3] Патент 2511922 (РФ) от 10.04.14 г, МПК F25B21/02. Термоэлектрический блок охлаждения / Деревянко В. А., Гладущенко В.Н., Гейнц Э. Р., Коков Е. Г., Васильев Е.Н., Руссков В. В. [Patent 2511922 (RF), dated 10.04.14, MPK F25B21/02. Thermoelectric cooling unit. / Derevjanko V.A., Gladushchenko V.N., Gejnts E.R., Kokov E. G., Vasil'ev E.N., Russkov V. V. (in Russian)].

[4] Васильев Е.Н., Гейнц Э. Р., Деревянко В. А., Коков Е. Г., Кукушкин С. В. Термоэлектрический блок охлаждения. Журн. Сиб. федер. ун-та. Техника и технологии, 2019, 12(2), 146-152. [Vasil'ev E.N., Gejnts E.R., Derevyanko V.A., Kokov E. G., Kukushkin S. V. Thermoelectric cooling block, J. Sib. Fed. Univ. Eng. technol., 2019, 12(2), 146-152 (in Russian)].

[5] Васильев Е.Н. Термоэлектрическое охлаждение теплонагруженных элементов электроники. Микроэлектроника. 2020, 49(2), 133-141. [Vasil'ev E.N., Thermoelectric cooling of heatloaded electronics, Russian Microelectronics., 2020, 49(2), 123-131].

[6] Васильев Е.Н. Определение режимов термоэлектрического охлаждения теплонагруженных элементов электроники. Микроэлектроника. 2020, 49(4), 297-303 [Vasil'ev E.N., Determination of thermoelectric cooling modes of heat-loaded electronics, Russian Microelectronics, 2020, 49(4), 278-284].

[7] Васильев Е.Н. Влияние термических сопротивлений на холодильный коэффициент термоэлектрической системы охлаждения. Журнал технической физики, 2021, 91(5), 743-747 [Vasil'ev E.N. Effect of thermal resistances on the coefficient of performance of a thermoelectric cooling system. Technical Physics, 2021, 66(5), 720-724].

[8] Васильев Е.Н. Расчет термического сопротивления теплораспределителя системы охлаждения теплонагруженного элемента. Журнал технической физики, 2018, 88(4), 487-491 [Vasil'ev E. N. Calculation of the Thermal Resistance of a Heat Distributer in the Cooling System of a Heat-Loaded Element. Technical Physics, Tech. Phys. 2018, 63(4), 471-475].

[9] Васильев Е.Н. Моделирование теплообмена в ребрах охлаждения. Журн. Сиб. федер. ун-та. Техника и технологии, 2020, 13(6), 669-676 [Vasil'ev E. N. Heat Exchange Modeling in Cooling Fins. J. Sib. Fed. Univ. Eng. technol. 2020, 13(6), 669-676 (in Russian)].

$$
-423-
$$

